Original Paper

\title{
The importance of IDH1, ATRX AND WT- 1 MUTATIONS IN GLIOBLASTOMA
}

\author{
Gülsün Gülten ${ }^{1}$, Nagihan Yalçin ${ }^{1}$, Bahar Baltalarli ${ }^{2}$, Gamze GökÖz DoĞu³, Feridun Acar ${ }^{4}$, \\ YÜCEL DOĞRUEL ${ }^{4}$
}

${ }^{1}$ Pathology Department, Pamukkale University Faculty of Medicine, Denizli, Turkey

${ }^{2}$ Radiation Oncology Department, Pamukkale University Faculty of Medicine, Denizli, Turkey

${ }^{3}$ Medical Oncology Department, Pamukkale University Faculty of Medicine, Denizli, Turkey

${ }^{4}$ Brain Surgery Department, Pamukkale University Faculty of Medicine, Denizli, Turkey

\begin{abstract}
Numerous genetic pathways associated with glioblastoma development have been identified. In this study, we investigated the prognostic significance of IDH1 and ATRX mutations and WT- 1 and p53 expression in glioblastomas and that of surgical methods, radiotherapy and chemotherapy. 83 patients with glioblastomas were retrospectively evaluated. Immunohistochemical analysis was performed for IDH1, ATRX and WT-1 expression. Tumour cells were positive for IDH1 in $9.6 \%$ of the patients. In $4.8 \%$ of the patients, loss of ATRX expression was observed in tumour cells; $86.7 \%$ of the patients were WT-1 positive, and $12.05 \%$ of the patients were $\mathrm{p} 53$ positive. No statistically significant difference was found in the progression-free and overall survival according to IDH1, ATRX, WT-1 and p53 expression. There was a statistically significant difference in the progression-free and overall survival according to the radiotherapy status. There was a statistically significant difference in the overall survival according to the chemotherapy status. There was no statistically significant difference in the progression-free and overall survival according to the surgical method. IDH1 and ATRX mutations, p53 overexpression and WT-1 expression alone did not have a significant effect on the prognosis of patients with glioblastoma; however, radiotherapy and chemotherapy had a positive effect on survival.
\end{abstract}

Key words: glioblastoma, immunohistochemistry, IDH1, ATRX, WT-1.

\section{Introduction}

Glioblastomas are the most malignant brain tumours, constituting $45-50 \%$ of primary malignant brain tumours [1]. In glioblastoma, the mean survival is 15 months, with a combination of maximal safe surgical resection, adjuvant radiotherapy and concurrent adjuvant temozolomide treatment [2]. Usually, patients with tumours respond poorly to radiotherapy and chemotherapy [3]. Therefore, there is a need for developing new therapeutic approaches for glioblastomas. The most promising treatment approach is the identification of genetic pathways leading to the development of glioblastomas [4]. In study conducted in 2008, it was found that IDH1/2 mutations played a role in the genetic pathways leading to glioblastoma formation [5]. IDH mutations occurred in the early stages of tumourigenesis, affected glial precursor cells and were acquired before TP53 mutations and 1p/19q co-deletion [6]. In the 2016 WHO classification, glioblastomas have been classified according to molecular markers such as IDH mutations 
or $1 \mathrm{p} / 19 \mathrm{q}$ deletion [7]. IDH1/2 mutation states play a role in the classification of diffuse gliomas [5]. Mutations of IDH2 are less frequent than those of IDH1. The frequency of IDH1 mutations is low in primary glioblastomas; however, it is seen in $60-80 \%$ of secondary glioblastomas developing from astrocytomas or oligodendroglial tumours [8]. IDH-mutant gliomas have a better prognosis than IDH-wild type gliomas [7]. ATRX encodes a protein involved in the chromatin rearrangement pathway, allowing the histone $\mathrm{H} 3.3$ to be incorporated into heterochromatin [9]. ATRX mutations occur in approximately $57 \%$ of secondary glioblastomas, but they is rare in primary glioblastomas. In glioblastomas, ATRX mutations are often accompanied by IDH1 and TP53 mutations [10]. ATRX mutations are a good prognostic factor $[11,12]$. Wilm's tumour (WT-1) gene is a tumour suppressor gene encoding the protein that acts as a transcription factor involved in cell growth and differentiation [13]. WT-1 plays a role in gliomagenesis and it is overexpressed in astrocytic tumours; it is correlated with the grade and $\mathrm{Ki}-67$ proliferation index. High WT-1 levels may be caused by cellular proliferation $[14,15]$. In a study by Cancer Genome Atlas, genetic changes in the p53 pathway have been reported in $90 \%$ of glioblastomas [16]. TP53 mutation is an early genetic alteration leading to secondary glioblastoma formation $[16,17]$. The aim of this study was to investigate the prognostic significance of IDH1, ATRX, WT-1 and p53 expressions and that of surgical methods, radiotherapy and chemotherapy in patients with glioblastomas using immunohistochemical methods.

\section{Material and methods}

Overall, 83 patients investigated in the Pathology Department of Pamukkale University, Faculty of Medicine, between 2010 and 2016 and diagnosed with glioblastomas were included in the study and evaluated retrospectively. Haematoxylineosin-stained preparations for all subjects were prepared from formalin-fixed paraffin-embedded tissue samples, and all immunohistochemical preparations previously applied and kept in the archives were re-evaluated. The block that best reflected the tumour morphology was selected for each case, and immunohistochemical staining for WT-1, ATRX and IDH1 was performed on this block. P53 (Ventana, DO-7 clone, pre-diluted) immunohistochemistry preparations in the archives were re-evaluated. Dianova polyclonal antibody (clone H09, 1/20) was used for IDH1, Sigma-Aldrich polyclonal antibody (clone HPA001906, 1/100) was used for ATRX and polyclonal antibody (Ventana, clone $6 \mathrm{~F}-\mathrm{H} 2$, pre-diluted) was used for WT-1; 3- $\mu \mathrm{m}$ thick sections were obtained from formalin-fixed, paraffin-embedded tissue samples selected for immunohistochemical staining, placed on electrostatically charged slides and dried in an incubator at $60^{\circ} \mathrm{C}$ for at least 2 hours. The entire staining process, including deparaffinization and antigen release, was performed using the Ventana BenchMark LT fully automated machine.

Cytoplasmic staining was performed, and staining intensity in tumour cells was semiquantitatively evaluated for IDH1. Cases with widespread intense cytoplasmic staining in tumour cells were considered as "IDH1 positive" for its mutation [18], and those with no tumour cell staining were considered as 'IDH1 negative'. For ATRX evaluation, staining results of vascular endothelial cells and normal glial cells were considered as the internal positive control. Nuclear staining in tumour cells was evaluated and calculated as a percentage. The presence of nuclear ATRX staining in $<10 \%$ of tumour cells showed expression loss for ATRX and was considered as 'ATRX positive' for its mutation [19]. For WT-1evaluation, staining results of vascular endothelial cells were considered as the internal positive control. Cytoplasmic staining was evaluated in tumour cells. The cell percentage that was positive on staining was calculated. Staining in $>50 \%$ of tumour cells was considered as WT- 1 positive, and staining in $\leq 50 \%$ of tumour cells was considered as WT-1 negative. Strong nuclear staining in $\geq 80 \%$ tumour cells was considered as p53 overexpression and "p53 positive" mutation.

Information on surgical treatment was obtained from the Department of Neurosurgery. Stereotactic biopsy as well as gross total and subtotal resections were performed surgically for patients depending on tumour localization. Information on postoperative radiotherapy treatment was obtained from Radiation Oncology Department records and that on chemotherapy treatment, progression-free survival (PFS) and overall survival (OS) data was obtained from Medical Oncology Department records. Survival data from the day of first diagnosis until December 2017 were used to calculate prognosis.

\section{Statistical analysis}

All analyses were performed using the SPSS software (version 21.0, SPSS Inc., Chicago, IL, USA). Descriptive statistics were presented as number, percentage, mean and standard deviation, median and minimum and maximum values. OS and PFS were used to predict the prognosis of IDH1 and ATRX mutations and WT- 1 and $\mathrm{p} 53$ expression and to calculate the effect of treatment on prognosis. OS was defined as the time between the diagnosis and patient death or final follow-up. PFS was defined as the time between the diagnosis and relapse or final follow-up. Kaplan-Meier and log-rank tests were used for survival analysis. A p-value of $<0.05$ was considered statistically significant. 
Ethical approval was obtained for this study from Pamukkale University Non-invasive Clinical Research Ethics Committee (Dated 06/28/2016, No. 13).

\section{Results}

This study was conducted with 83 patients with glioblastomas who were selected from those diagnosed in the Pathology Department of Pamukkale University, Faculty of Medicine, between 2010 and 2016. The mean age of the 83 patients was 57.95 \pm 12.70 years, and the median age was 62 years; $49.4 \%(\mathrm{n}=41)$ of the patients were female and $50.6 \%(n=42)$ were male. The male/female ratio was $1.02 / 1$. In terms of histopathological classification $96.4 \%(n=80)$ of the patients were diagnosed with classic glioblastoma, $1.2 \%(\mathrm{n}=1)$ of the patients were diagnosed with giant cell glioblastomas and $2.4 \%(n=2)$ of the patients were diagnosed with gliosarcomas. According to clinical history, 95.2\% ( $\mathrm{n}=79$ ) of the patients had primary glioblastomas and $4.8 \%(n=4)$ had secondary glioblastomas. Three of the patients with secondary glioblastomas were previously diagnosed with diffuse astrocytoma, and 1 was diagnosed with gemistocytic astrocytoma registered in our pathology laboratory. The tumours were excised by gross total resection in $44.6 \%(\mathrm{n}=37)$ of the patients and by subtotal resection in $53 \%(n=44)$ of the patients. Owing to tumour localization, diagnostic stereotactic biopsy was performed in $2.4 \%(\mathrm{n}=2)$ of the patients; $83.1 \%(n=69)$ of the patients received radiotherapy (60 Gray dose) five days a week for 6 weeks after surgery, and $16.9 \%(\mathrm{n}=14)$ of the patients could not receive radiotherapy owing to poor general condition. Temozolomide $\left(75 \mathrm{mg} / \mathrm{m}^{2} /\right.$ day) was concurrently administered to the patients receiving radiotherapy. After radiotherapy, 67.5\% ( $\mathrm{n}=56)$ of the patients received 6 courses of $150-200 \mathrm{mg} /$ $\mathrm{m}^{2}$ of temozolomide treatment for 5 days once every 28 days in the Oncology Clinic; $32.5 \%(n=56)$ of the patients could not receive treatment owing to poor general condition. Based on available data until December $2017,7.2 \%(n=6)$ of the patients survived, and $92 \%$ ( $\mathrm{n}=77$ ) of the patients died. The distribution of patients according to clinical findings is shown in Table I.

\section{Immunohistochemical findings in glioblastoma}

Immunohistochemical results for IDH-1, ATRX, WT- 1 and p53 staining are shown in Table II. 90.4\% $(\mathrm{n}=75)$ of 83 patients were IDH1 negative. Cytoplasmic IDH1 staining in tumour cells was observed in $9.6 \%(\mathrm{n}=8)$ of the patients (Figs. 1A, B), and 95.2\% ( $\mathrm{n}=79$ ) of the patients had nuclear ATRX staining in $\geq 10 \%$ of tumour cells and were considered as "no ATRX mutation". More than $90 \%$ nuclear expression loss in tumour cells was observed in $4.8 \%(\mathrm{n}=4)$ of the patients, and these were considered as "ATRX mutation" (Figs. 1C, D). WT-1 staining ranged from $5 \%$ to $98 \%$, with a mean staining percentage of $68.37 \% \pm 20.071 \%$ and a median of $70 \%$. The percentage of WT-1 staining was $>50 \%$ in $86.7 \%(n=72)$ of the patients and were evaluated as WT- 1 positive. The percentage of WT-1 staining was $\leq 50 \%$ in $13.3 \%(\mathrm{n}=11)$ of the patients and were evaluated as WT-1 negative (Figs. 2A, B). The mean p53 staining percentage was $28.63 \pm 28.921 \%$, and the median value was $15 \%$. p53 staining percentage was $\geq 80 \%$ in $12.05 \%(\mathrm{n}=10)$ of the patients and were evaluated as positive. p53 staining percentage was $<80 \%$ in $87.95 \%(\mathrm{n}=73$ ) of the patients and were evaluated as p53 negative (Figs. 2C, D).

Table I. The distribution of patients according to clinical findings

\begin{tabular}{|c|c|}
\hline PARAmeter & \\
\hline \multicolumn{2}{|l|}{ Age } \\
\hline Median age & $57.95 \pm 12.70(62)$ \\
\hline Range & $21-83$ \\
\hline \multicolumn{2}{|l|}{ Sex } \\
\hline Male & $42(50.6 \%)$ \\
\hline Female & $41(49.4 \%)$ \\
\hline Male/female & $1.02 / 1$ \\
\hline \multicolumn{2}{|c|}{ Histopathological classification } \\
\hline Classical glioblastoma & $80(96.4 \%)$ \\
\hline Giant cell glioblastoma & $1(1.2 \%)$ \\
\hline Gliosarcoma & $2(2.4 \%)$ \\
\hline \multicolumn{2}{|c|}{ According to the clinical history } \\
\hline Primary glioblastoma & $79(95.2 \%)$ \\
\hline Secondary glioblastoma & $4(4.8 \%)$ \\
\hline \multicolumn{2}{|l|}{ Surgery } \\
\hline Gross total resection & $37(44.6 \%)$ \\
\hline Subtotal resection & $44(53 \%)$ \\
\hline Stereotactic biopsy & $2(2.4 \%)$ \\
\hline \multicolumn{2}{|l|}{ Radiotherapy } \\
\hline Receive & $69(83.1 \%)$ \\
\hline Not receive & $14(16.9 \%)$ \\
\hline \multicolumn{2}{|l|}{ Chemotherapy } \\
\hline Receive & $56(67.5 \%)$ \\
\hline Not receive & $27(32.5 \%)$ \\
\hline \multicolumn{2}{|l|}{ Survival } \\
\hline Exitus & $77(92 \%)$ \\
\hline Alive & $6(7.2 \%)$ \\
\hline
\end{tabular}


Table II. IDH1, ATRX, WT-1 and p53 immunohistochemical results and survival analysis

\begin{tabular}{|c|c|c|c|c|c|c|}
\hline Glioblastom & $\mathbf{N}$ & $\%$ & $\begin{array}{c}\text { MEDIAN } \\
\text { PFS, MONTHS }\end{array}$ & P-value & $\begin{array}{c}\text { MEDIAN } \\
\text { OS, MONTHS }\end{array}$ & P-value \\
\hline \multicolumn{7}{|l|}{ IDH1 } \\
\hline Positive & 8 & 9.6 & 5 & 0.217 & 11 & 0.297 \\
\hline Negative & 75 & 90.4 & 4 & & 8 & \\
\hline \multicolumn{7}{|l|}{ ATRX } \\
\hline Positive & 79 & 95.2 & 15 & 0.214 & 15 & 0.342 \\
\hline Negative & 4 & 4.8 & 4 & & 8 & \\
\hline \multicolumn{7}{|l|}{ WT1 } \\
\hline Positive & 72 & 86.7 & 10 & 0.800 & 15 & 0.454 \\
\hline Negative & 11 & 13.3 & 2 & & 11 & \\
\hline \multicolumn{7}{|l|}{ P53 } \\
\hline Positive & 10 & 12.05 & 4 & 0.697 & 6 & 0.798 \\
\hline Negative & 73 & 87.95 & 5 & & 8 & \\
\hline
\end{tabular}
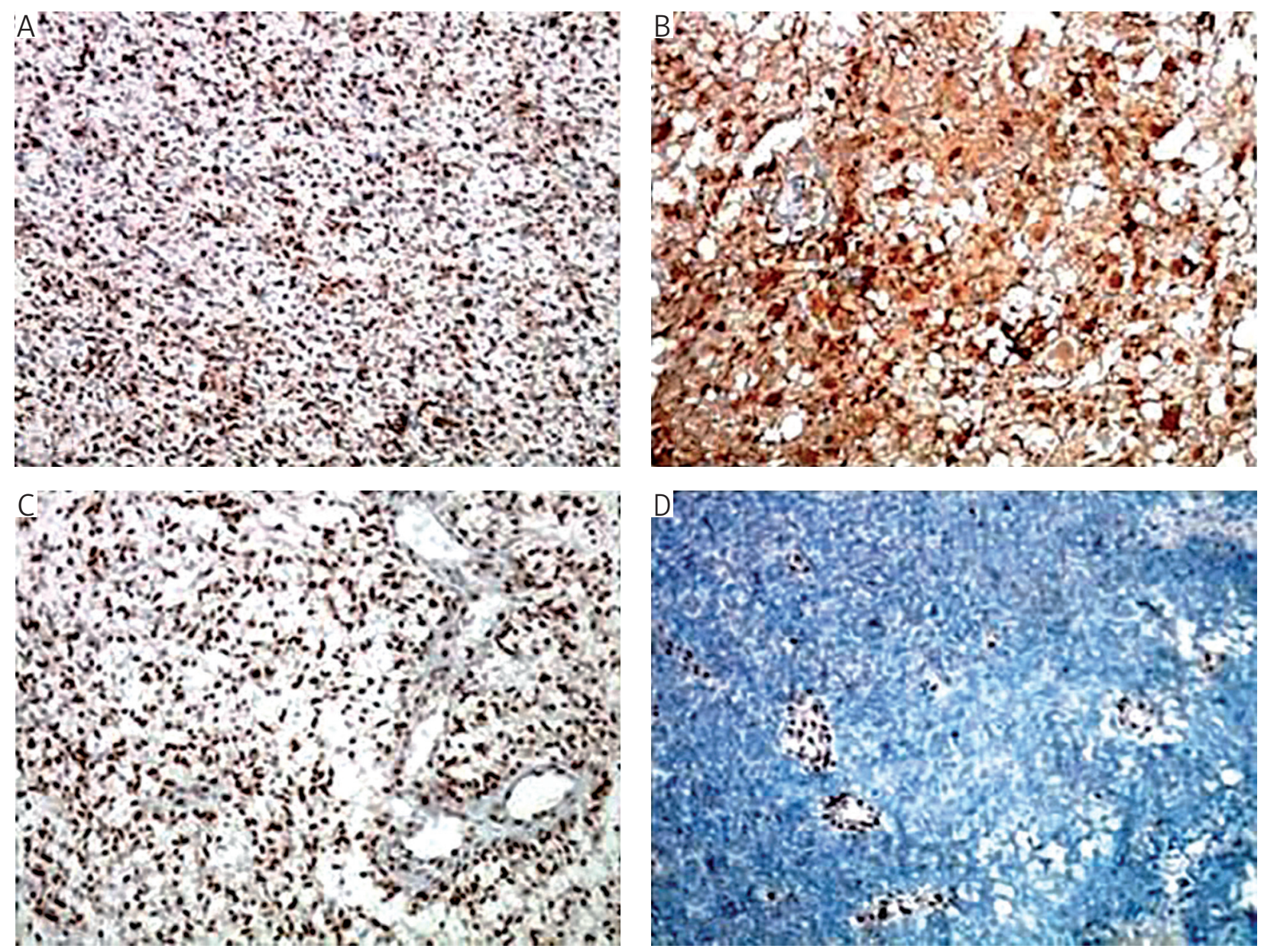

Fig. 1. Glioblastoma cases. A, B) Strong cytoplasmic IDH1 positivity in glioblastoma (IDH1, 200×). C) ATRX positivity in glioblastoma (ATRX, 200×). D) ATRX negativity in glioblastoma, endothelial cells positive as internal control (ATRX, 200×) 

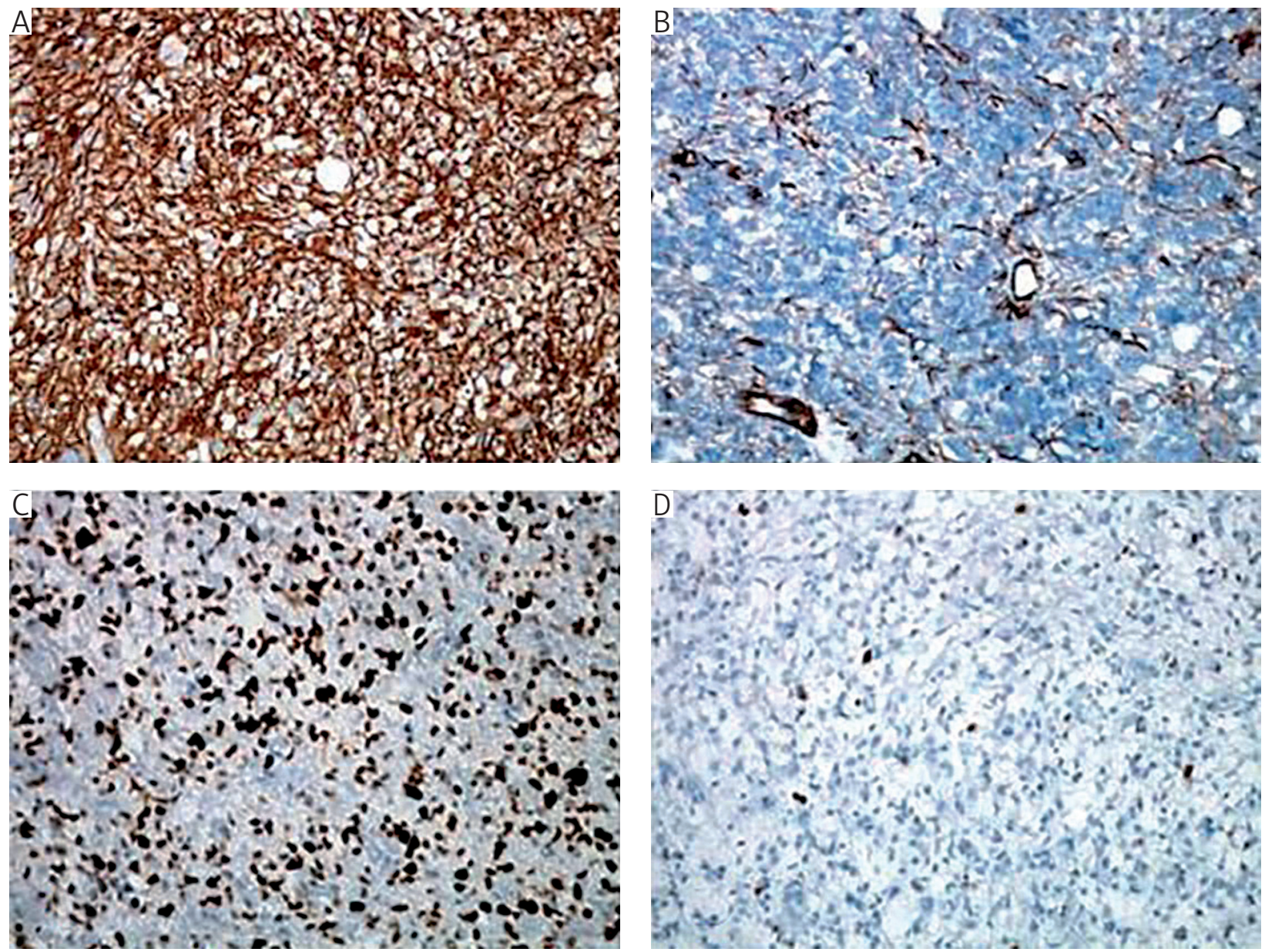

Fig. 2. Glioblastoma cases. A) WT-1 positivity in glioblastoma (WT-1, 200×). B) WT-1 negativity in glioblastoma (WT-1, $200 \times$ ). C) P53 positivity in glioblastoma (P53, 200×). D) P53 negativity in glioblastoma (P53, 200×)

\section{Survival findings}

Overall, $7.2 \%(n=6)$ of 83 patients were alive as of December 2017; and 92.8\% ( $\mathrm{n}=77)$ of the patients died. The mean PFS was $8.994 \pm 1.321$ months, and the median PFS was 5 months. The mean OS was $11.878 \pm 1.364$, and the median OS was 8 months. Survival analysis according to the expression states of IDH-1, ATRX, WT- 1 and $\mathrm{p} 53$ is shown in Table II. Survival analysis based on radiotherapy, chemotherapy and surgery is shown in Table III.

Survival analysis based on IDH1

The mean PFS in IDH1-positive patients was $13.375 \pm 4.950$ months (median: 5 months) and that in IDH1-negative patients was $8.255 \pm 1.257$ months (median: 4 months). The mean OS in IDH1-positive patients was $15.250 \pm 4.616$ months (median: 11 months) and that in IDH1-negative patients was $11.298 \pm 1.345$ months (median: 8 months). There was no statistically significant difference in PFS and OS according to IDH1 expression ( $\mathrm{p}=0.217$ and $\mathrm{p}=0.297$, respectively; Figs. 3A, B).

\section{Survival analysis based on ATRX}

In patients with loss of ATRX expression, the mean PFS was $15.5 \pm 3.279$ months (median: 15 months), and the mean OS was $17.25 \pm 2.955$ months (median: 15 months). In patients without any loss of ATRX expression, the mean PFS was $8.752 \pm 1.397$ months (median: 4 months), and the mean OS was $11.662 \pm 1.434$ months (median: 8 months). There was no statistically significant difference in PFS and OS according to ATRX expression $(\mathrm{p}=0.214$ and $\mathrm{p}=0.342$, respectively; Figs. 3C, D).

\section{Survival analysis based on WT-1}

The mean PFS in WT-1-positive patients was $8.627 \pm 1.287$ months (median: 5 months) and that in WT-1-negative patients was $9.182 \pm 3.396$ months (median: 3 months). The mean OS in WT-1-positive patients was $11.407 \pm 1.389$ months (median: 7 months) and that in WT-1-negative patients was $13.091 \pm 3.174$ months (median: 12 months). There was no statistically significant difference in PFS and OS according to WT- 1 expression $(\mathrm{p}=0.800$ and $\mathrm{p}=0.454$, respectively; Figs. 3E, F). 
Table III. Survival analysis based on radiotherapy, chemotherapy and surgery

\begin{tabular}{|c|c|c|c|c|c|c|}
\hline GLIOBLASTOMA & $\mathbf{N}$ & $\%$ & $\begin{array}{c}\text { MEDIAN } \\
\text { PFS, MONTHS }\end{array}$ & P-Value & $\begin{array}{c}\text { MEDIAN } \\
\text { OS, MONTHS }\end{array}$ & P-VAlue \\
\hline \multicolumn{7}{|l|}{ RT } \\
\hline Receive & 69 & 83.1 & 6 & 0.000 & 11 & 0.000 \\
\hline Not receive & 14 & 16.9 & 1 & & 1 & \\
\hline \multicolumn{7}{|l|}{ KT } \\
\hline Receive & 56 & 67.5 & 6 & 0.079 & 12 & 0.006 \\
\hline Not receive & 27 & 32.5 & 2 & & 2 & \\
\hline \multicolumn{7}{|l|}{ Surgery } \\
\hline Gross total resection & 37 & 44.6 & 4 & 0.983 & 12 & 0.516 \\
\hline Subtotal resection & 44 & 53 & 5 & & 6 & \\
\hline
\end{tabular}

\section{Survival analysis based on P53}

The mean PFS in patients with $\mathrm{p} 53$ overexpression was $11.000 \pm 4.297$ months (median: 4 months). The mean PFS in patients without p53 overexpression was $8.666 \pm 1.394$ months (median: 5 months). The mean OS in patients with $\mathrm{p} 53$ overexpression was $13.000 \pm 4.142$ months (median: 6 months). The mean OS in patients without $\mathrm{p} 53$ overexpression was $11.583 \pm 1.409$ months (median: 8 months). There was no statistically significant difference in PFS and OS according to P53 overexpression ( $\mathrm{p}=0.697$ and $p=0.798$, respectively; Figs. $3 \mathrm{G}, \mathrm{H}$ ).

\section{Survival analysis based on radiotherapy}

The mean PFS was $1.429 \pm 0.173$ months (median: 1 month) in patients who did not receive radiotherapy and $10.529 \pm 1.524$ months (median: 6 months) in patients who received radiotherapy. There was a statistically significant difference in the PFS based on radiotherapy status $(\mathrm{p}=0.000$; Fig. 4A). The mean OS was $1.429 \pm 0.173$ months (median: 1 month) in patients who did not receive radiotherapy and $13.998 \pm 1.518$ months (median: 11 months) in patients who received radiotherapy. There was a statistically significant difference in OS based on their radiotherapy status $(\mathrm{p}=0.000$; Fig. 4B).

\section{Survival analysis based on chemotherapy}

There was no statistically significant difference in the PFS based on chemotherapy status $(\mathrm{p}=0.079$; Fig. 4C). The mean OS was $8.963 \pm 3.091$ months (median: 2 months) in patients who did not receive chemotherapy and $13.317 \pm 1.286$ months (median: 12 months) in patients who received chemotherapy. There was a statistically significant difference in OS based on chemotherapy status ( $p=0.006$; Fig. 4D).

\section{Survival analysis based on surgical method}

Two patients who underwent stereotactic biopsy were excluded from the evaluation owing to the small sample size. Of the 37 patients who underwent gross total resection, 91.9\% $(\mathrm{n}=34)$ died. Of the $44 \mathrm{pa}-$ tients who underwent subtotal resection, $93.2 \%$ $(\mathrm{n}=41)$ died. There was no statistically significant difference in PFS and OS based on the surgical meth$\operatorname{od}(\mathrm{p}=0.983, \mathrm{p}=0.516$, respectively; Figs. 4E, F).

\section{Discussion}

Owing to recently developed molecular techniques, important biomarkers have been found for the diagnosis and prognosis of glioblastomas. These markers provide valuable information about the pathogenesis of gliomas and have become the target for new therapeutic approaches [20]. In this study, we evaluated the expression and prognostic significance of IDH1, ATRX, WT- 1 and $\mathrm{p} 53$ in patients with glioblastomas and the prognostic significance of surgical methods, radiotherapy and chemotherapy on their survival.

DNA sequencing methods, fluorescence in situ hybridisation and pyrosequencing methods have been used to detect IDH mutations in patients with glioblastomas [21]. Capper et al. compared the DNA sequencing method and immunohistochemical method for detecting IDH1 mutations in 186 patients with gliomas. Using R132H-mutation specific antibodies, they determined the sensitivity and specificity of the immunohistochemical method to be $94 \%$ and $100 \%$, respectively. They reported that immunohistochemical methods could be used as a standard procedure owing to the difficulty associated with genetic analysis methods such as DNA sequencing [22]. In studies in which immunohistochemical methods were performed, Popova et al. detected IDH1 mutations in $11 \%$ patients with glioblastomas [23], and Chaurasia 

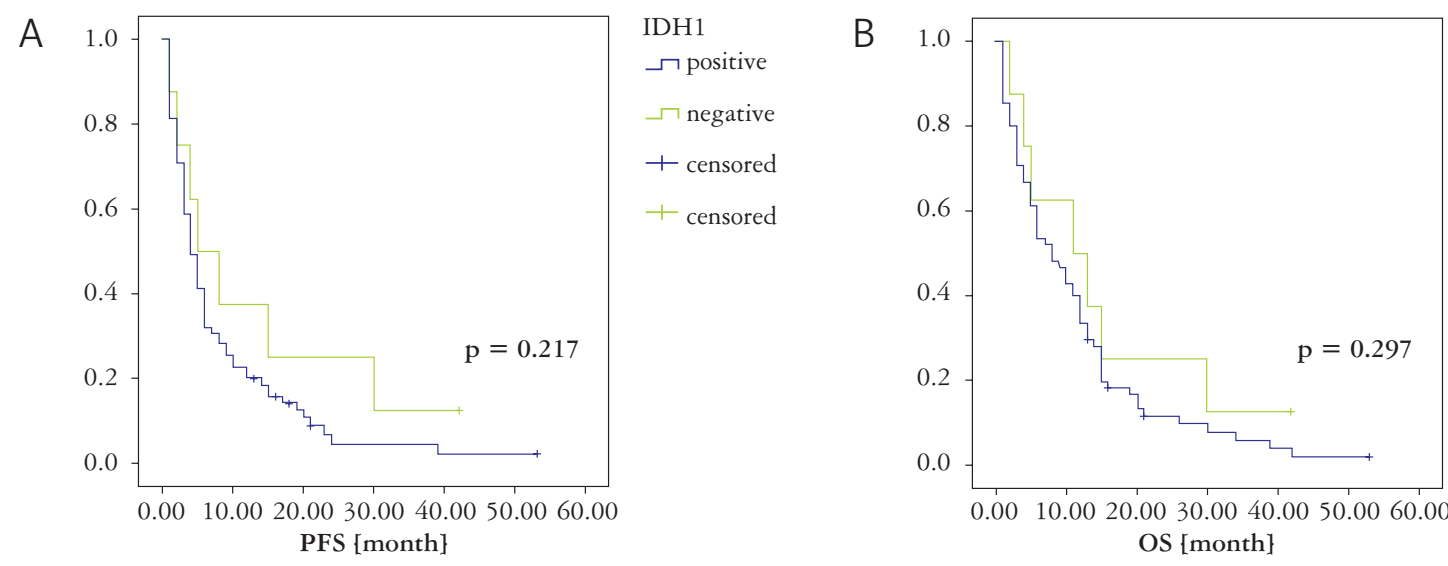

IDH1

$\neg$ positive

$\neg$ negative

+ censored

+ censored

C

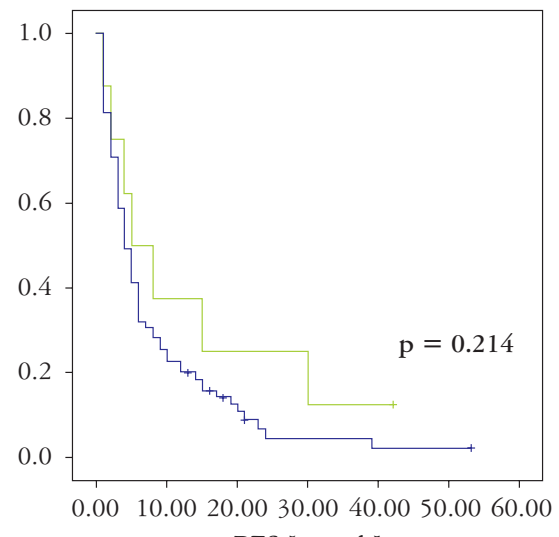

PFS [month]

E

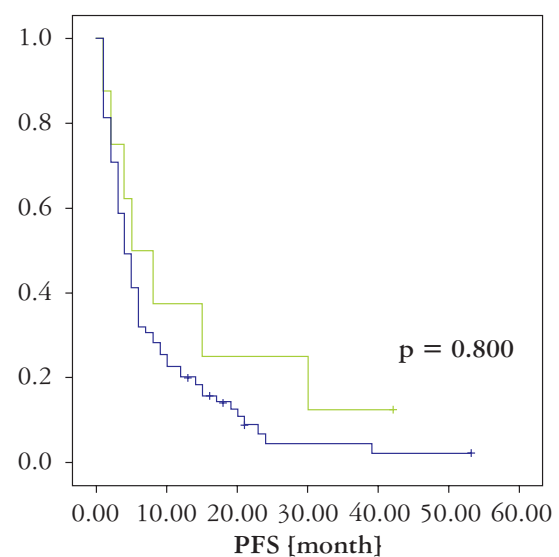

G

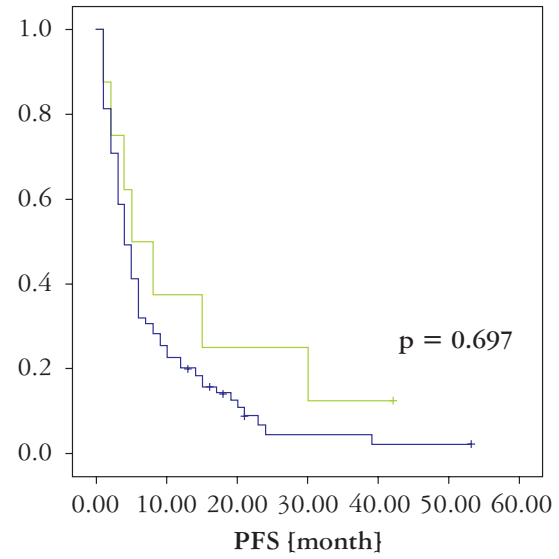

ATRX

$\neg$ positive

$\neg$ negative

+ censored

+ censored

WT-1

$\neg$ negative

$\neg$ positive

+ censored

+ censored

p53

$\neg$ negative

$\neg$ positive

+ censored

+ censored
D

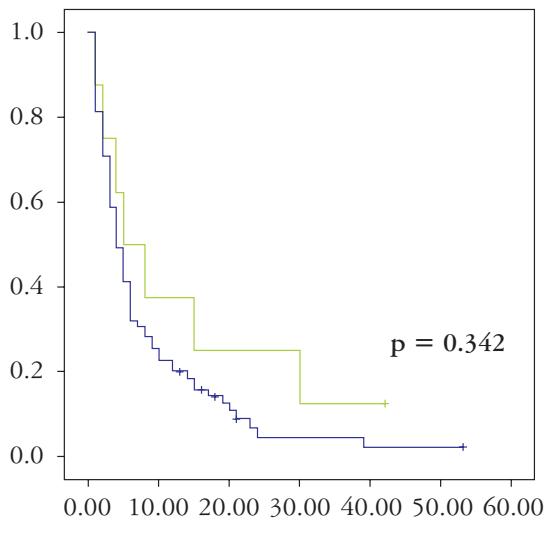

OS [month]

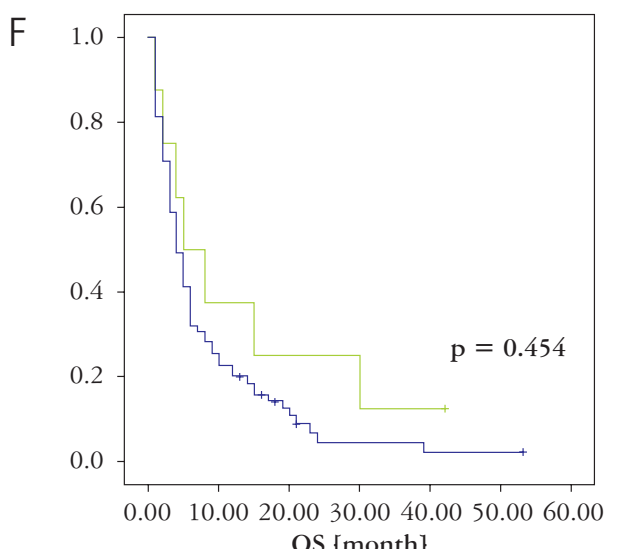

WT-1

$\neg$ negative

$\neg$ positive

+ censored

+ censored
$\mathrm{H}$

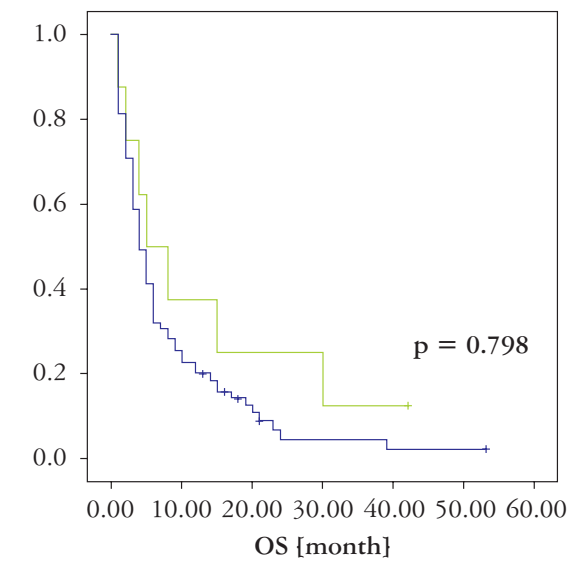

p53

$\neg$ negative

$\neg$ positive

+ censored

+ censored

Fig. 3. Kaplan-Meier curves. Progression-free survival (PFS) and overall survival rate (OS) for patients with glioblastoma. A) PFS according to IDH1 expression. B) OS according to IDH1 expression. C) PFS according to ATRX expression. D) OS according to ATRX expression. E) PFS according to WT-1 expression. F) OS according to WT-1 expression. G) PFS according to p53 expression. H) OS according to p53 expression. P-values were calculated by the log-rank test 
A

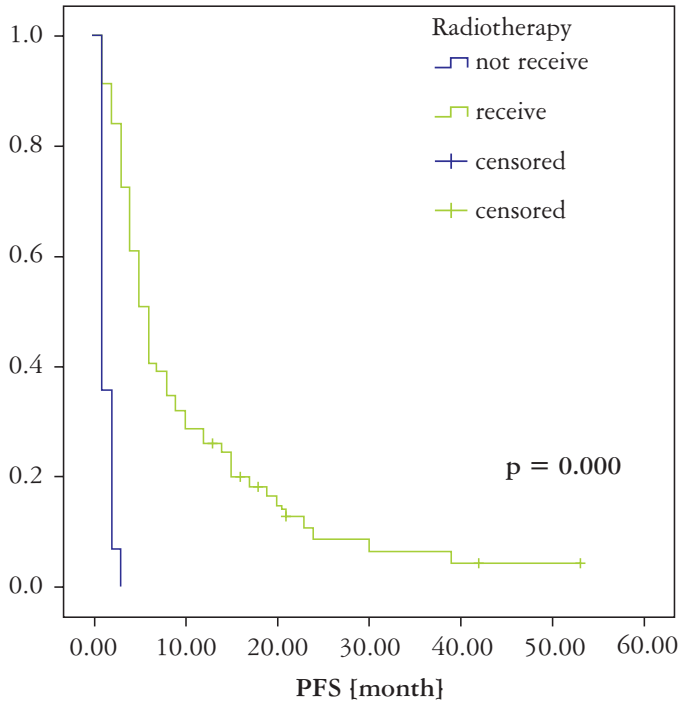

C

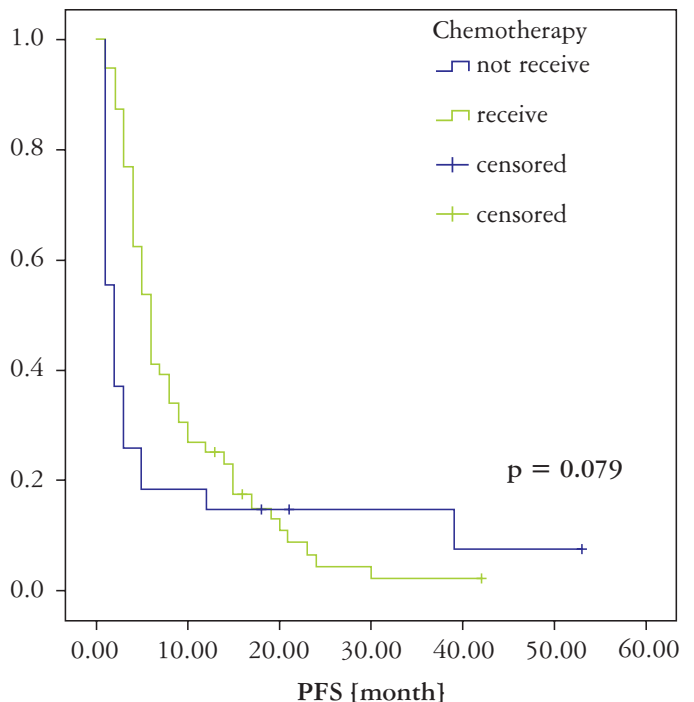

$\mathrm{E}$

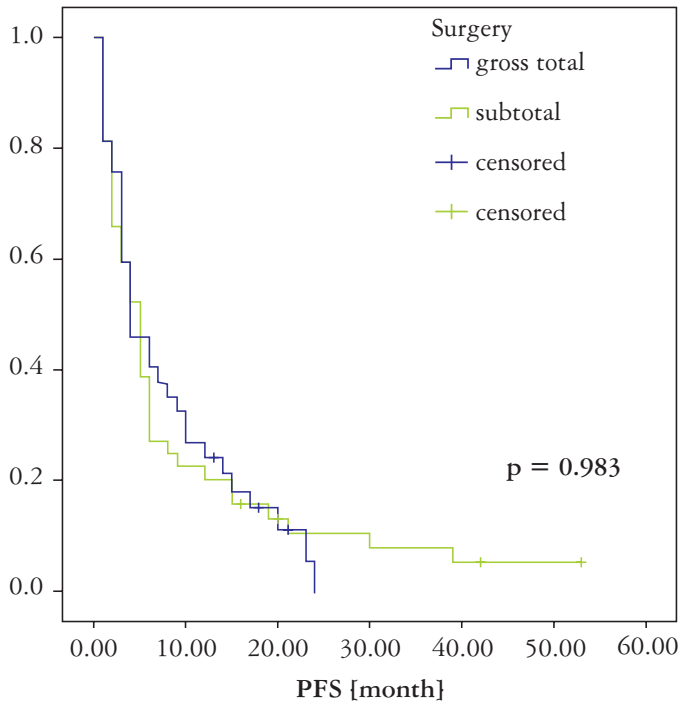

B

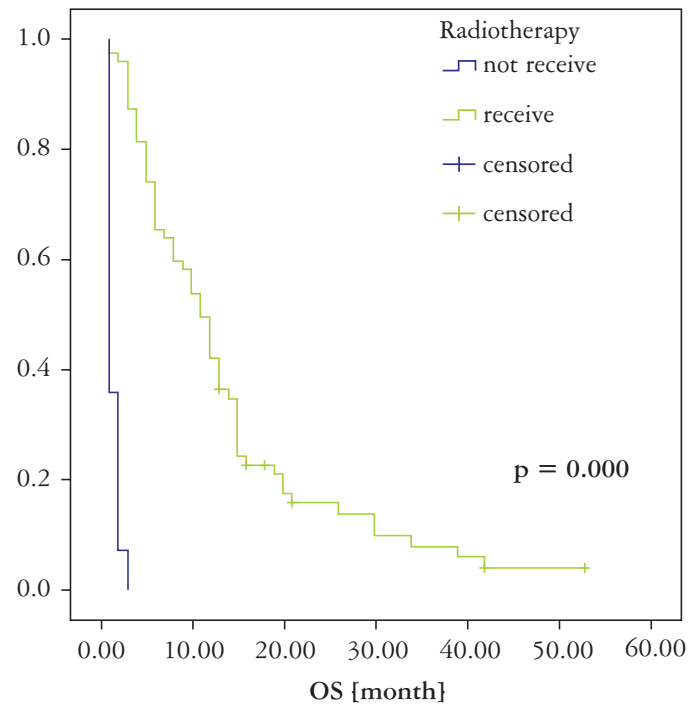

D

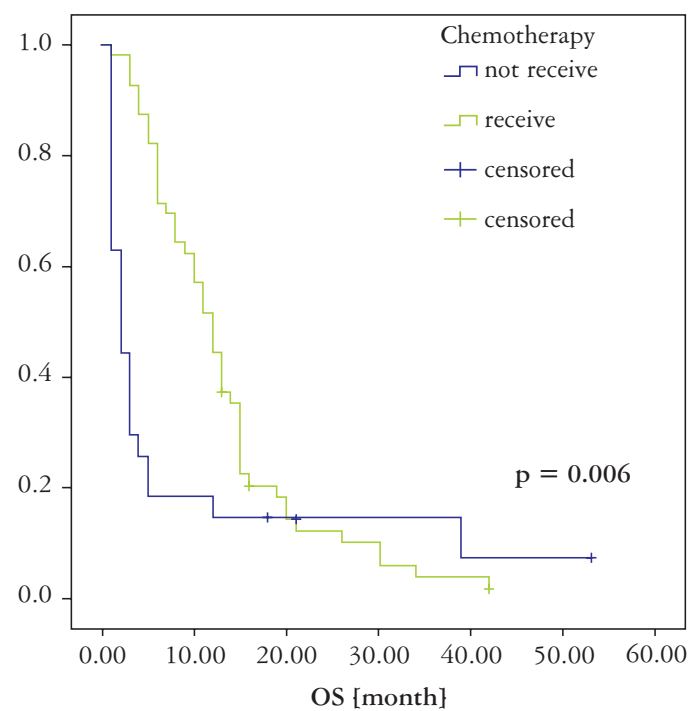

F

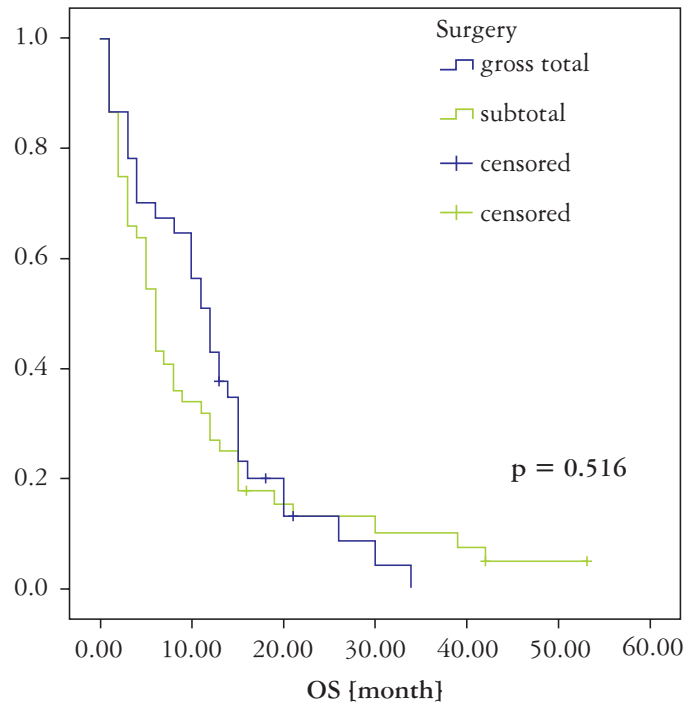

Fig. 4. Kaplan-Meier curves. Progression-free survival (PFS) and overall survival rate (OS) for patients with glioblastoma. A) PFS according to radiotherapy. B) OS according to radiotherapy. C) PFS according to chemotherapy. D) OS according to chemotherapy. E) PFS according to surgery. F) OS according to surgery. P-values were calculated by the log-rank test 
et al. detected IDH1 mutations in $10.4 \%$ patients with glioblastomas [11]. Pekmezci et al. conducted DNA sequencing and immunohistochemistry methods and detected IDH mutations in $14 \%$ of $360 \mathrm{pa}-$ tients with glioblastomas [24]. In the present study, we detected IDH1 mutations in $9.6 \%$ of 83 patients with glioblastomas by immunohistochemical methods. Our results are consistent with those of other studies in which immunohistochemical methods were performed.

IDH1 mutations are a good prognostic marker [5, 8, 25]. Glioblastomas with a IDH1 mutation had a better prognosis than anaplastic astrocytoma without any IDH1 mutation [25]. Kim et al. reported that IDH1/2 mutations had no prognostic value in low-grade gliomas [26]. In a meta-analysis, Chen et al. evaluated the prognostic value of IDH1/2 mutations and examined 15 studies for OS and 10 studies for PFS. They found that IDH1/2 mutations were associated with longer OS and PFS in patients with glioblastomas [27]. Combs et al. investigated patients with primary glioblastomas and found that OS was significantly longer in patients with IDH1 mutations and that there was a significant difference in the OS; however, they did not observe a significant difference in the PFS between patients with IDH mutants and IDH-wild type primary glioblastomas [28]. Paldor et al. compared 21 patients with IDH-mutant glioblastomas and 21 with IDH-wild type glioblastomas in terms of OS and PFS and found no statistically significant difference. They reported that IDH mutations did not provide a better prognosis of glioblastomas [29]. In the present study, although the mean as well as median PFS and OS were longer in patients with IDH mutations than those with wild type IDH, there was no statistically significant difference.

Although ATRX mutations are common in diffuse astrocytoma, they are rarely seen in oligoastrocytomas, oligodendrogliomas or glioblastomas. Immunohistochemical assessment of the loss of ATRX expression captures the majority of ATRX mutations and that the use of immunohistochemical tests for gliomas is highly reliable $[10,30]$. Loss of ATRX expression has been examined in various studies by immunohistochemical methods. Reuss et al. reported a loss of ATRX expression in 18\%, Liu et al. in $26 \%$ and Chaurasia et al. in $15.3 \%$ of patients with glioblastomas [11, 31, 32]. In the present study, loss of ATRX expression was detected in only 4 of 83 patients $(4.8 \%)$ and was evaluated as ATRX mutation.

Chaurasia et al. and Cai et al. found that ATRX mutation in glioblastomas had a statistically significant effect on survival. They found that ATRX mutation was a good prognostic factor [11, 12]. Pekmezci et al. did not observe a significant difference in survival with respect to ATRX mutation status in IDH-mutant glioblastomas; however, the presence of ATRX mutation in IDH-wild type glioblastomas was associated with better survival [24]. Uppar et al. reported that ATRX, IDH and p53 biomarkers did not affect prognosis in paediatric patients with glioblastomas [33]. In the present study, we found that the mean PFS and OS were longer in patients with ATRX mutation than in those without ATRX mutation. However, we did not find any statistically significant difference in PFS and OS in our patients.

WT-1 inhibits p53-mediated apoptosis, stimulates tumour cell proliferation and increases cellular longevity [34]. WT-1 plays a role in gliomagenesis and is expressed in astrocytic tumours. WT- 1 expression is correlated with the tumour grade $[14,15,35,36]$. Studies have demonstrated high WT-1 expression in glioblastomas $[15,37]$. Bourne et al. reported WT-1 expression in all cases of glioblastomas and found $\geq 20 \%$ WT1 expression in 36 out of 38 cases [38]. Consistent with the literature, we observed WT-1 expression in all of our patients with glioblastomas and found $\geq 20 \%$ WT- 1 staining in $96.4 \%$ of our patients.

Rauscher et al. and Schwab et al. showed that WT-1 expression was associated with poor prognosis in patients with astrocytic tumours. Schwab et al. showed that WT-1 expression decreased significantly in the presence of IDH1 mutation and loss of ATRX expression [39, 40]. There are few studies investigating survival with respect to the WT-1 expression status in patients with glioblastomas. Camacho-Urkaray et al. found a significantly decreased survival in patients with glioblastomas with decreased WT-1 levels [41]. Rauscher et al. compared survival according to WT-1 expression in patients with glioblastomas and found no significant difference. They reported that WT-1 expression was not a prognostic factor in patients with glioblastomas [42]. Here, we investigated WT-1 expression only in patients with glioblastomas and did not find a significant difference in PFS and OS.

TP53 mutation is a genetic alteration that occurs early in patients with gliomas and is detected in majority of patients with low-grade diffuse astrocytomas. Its prevalence in anaplastic astrocytoma developing from diffuse astrocytomas and secondary glioblastomas is similar to that of diffuse astrocytoma [43]. There are conflicting reports on the effect of TP53 mutation on the prognosis in patients with glioblastomas. TP53 mutations are not associated with the prognosis [44, 45]. Conversely, Schmidt et al. and Ohgaki et al. found that TP53 mutations were a good prognostic factor in patients with glioblastomas [37, 46].

Chaurasia et al. examined 163 patients with glioblastomas by immunohistochemical methods and found better PFS in p53-negative patients; however, there was no significant difference in the OS [11]. 
Montgomery et al. observed a shorter life expectancy at high p53 levels and reported that $\mathrm{p} 53$ was a poor prognostic factor [47]. Ogura et al. reported that no significant difference was found in the survival with respect to $\mathrm{p} 53$ expression in patients with glioblastomas [18]. In the present study, we did not find a significant difference in PFS and OS with respect to p53 overexpression.

Stupp et al. compared survival in patients with glioblastomas who received only radiotherapy and those who received radiotherapy and subsequent adjuvant temozolomide treatment after surgery. They found the median survival time to be 14.6 months in the latter compared with a median survival time of 12.1 months in the former [2]. Ohgaki et al. found that patients undergoing surgery or patients receiving radiotherapy had a longer life expectancy [17]. Here, we compared the gross total and subtotal surgery in terms of PFS and OS in the patients included in our study but could not obtain statistically significant results. We found a statistically significant difference in PFS and OS with respect to the radiotherapy status. Consistent with the literature, we found that the survival of patients who received radiotherapy survived was longer. In the patients included in our study, the median OS was 2 months in those who did not receive adjuvant temozolomide treatment compared with patients who received adjuvant temozolomide treatment, for whom the median OS was 12 months. We observed a statistically significant difference in the OS with respect to chemotherapy status; however, we did not obtain significant results in PFS.

In conclusion, we found that IDH1 and ATRX mutations, p53 overexpression and WT-1 expression alone did not have a significant effect on the prognosis in patients with glioblastoma; however, radiotherapy and chemotherapy had a positive effect on their survival. These findings should be supported by future studies conducted on larger series of patients by molecular methods.

Pamukkale University provided financial support in the form of research funding. The sponsor had no role in the design or conduct of this research.

The authors declare no conflict of interest.

\section{References}

1. Ohgaki H, Kleihues P. Population-based studies on incidence, survival rates, and genetic alterations in astrocytic and oligodendroglial gliomas. J Neuropathol Exp Neurol 2005; 64: 479-489.

2. Stupp R, Hegi ME, Mason WP, et al. Effects of radiotherapy with concomitant and adjuvant temozolomide versus radiotherapy alone on survival in glioblastoma in a randomised phase III study: 5-year analysis of the EORTC-NCIC trial. Lancet Oncol 2009; 10: 459-466.
3. Masui K, Cloughesy TF, Mischel PS. Review: molecular pathology in adult high-grade gliomas: from molecular diagnostics to target therapies. Neuropathol Appl Neurobiol 2012; 38: 271-291

4. Aldape K, Zadeh G, Mansouri S, et al. Glioblastoma: pathology, molecular mechanisms and markers. Acta Neuropathol 2015; 129: 829-848.

5. Parsons DW, Jones S, Zhang X, et al. An integrated genomic analysis of human glioblastoma multiforme. Science 2008; 321: 1807-1812

6. Watanabe T, Nobusawa S, Kleihues P, et al. IDH1 mutations are early events in the development of astrocytomas and oligodendrogliomas. Am J Pathol 2009; 174: 1149-1153.

7. Louis DN, Perry A, Reifenberger G, et al. The 2016 World Health Organization Classification of Tumors of the Central Nervous System: a summary. Acta Neuropathol 2016; 131: 803-820.

8. Yan H, Parsons DW, Jin G, et al. IDH1 and IDH2 mutations in gliomas. N Engl J Med 2009; 360: 765-773.

9. Schwartzentruber J, Korshunov A, Liu XY, et al. Driver mutations in histone $\mathrm{H} 3.3$ and chromatin remodelling genes in pediatric glioblastoma. Nature 2012; 482: 226-231.

10. Jiao Y, Killela PJ, Reitman ZJ, et al. Frequent ATRX, CIC, FUBP1 and IDH1 mutations refine the classification of malignant gliomas. Oncotarget 2012; 3: 709-722.

11. Chaurasia A, Park SH, Seo JW, et al. Immunohistochemical analysis of ATRX, IDH1 and p53 in glioblastoma and their correlations with patient survival. J Korean Med Sci 2016; 31 : 1208-1214.

12. Cai J, Zhang C, Zhang W, et al. ATRX, IDH1-R132H and $\mathrm{Ki}-67$ immunohistochemistry as a classification scheme for astrocytic tumors. Oncoscience 2016; 3: 7-8.

13. Pritchard-Jones K. The Wilms tumour gene, WT-1, in normal and abnormal nephrogenesis. Pediatr Nephrol 1999; 13: 620-625.

14. Hashiba T, Izumoto S, Kagawa N, et al. Expression of WT1 protein and correlation with cellular proliferation in glial tumors. Neurol Med Chir 2007; 47: 165-170.

15. Oji Y, Suzuki T, Nakano Y, et al. Overexpression of the Wilms' tumor gene WT1 in primary astrocytic tumors. Cancer Sci 2004; 95: 822-827.

16. Cancer Genome Atlas Research Network. Comprehensive genomic characterization defines human glioblastoma genes and core pathways. Nature 2008; 455: 1061-1068.

17. Ohgaki H, Kleihues P. Genetic pathways to primary and secondary glioblastoma. Am J Pathol 2007; 170: 1445-1453.

18. Ogura R, Tsukamoto Y, Natsumeda M, et al. Immunohistochemical profiles of IDH-1, MGMT and P53: Practical significance for prognostication of patients with diffuse gliomas. Neuropathology 2015; 35: 324-335.

19. Wiestler B, Capper D, Holland-Letz T, et al. ATRX loss refines the classification of anaplastic gliomas and identifies a subgroup of IDH mutant astrocytic tumours with better prognosis. Acta Neuropathol 2013; 126: 443-451.

20. Wen PY, Kesari S. Malignant gliomas in adults. N Engl J Med 2008; 359: 492-507.

21. Lee KS, Choe G, Nam KH, et al. Immunohistochemical classification of primary and secondary glioblastomas. Korean J Pathol 2013; 47: 541-548.

22. Capper D, Weissert S, Balss J, et al. Characterization of R132H mutation-specific IDH1 antibody binding in brain tumors. Brain Pathology 2010; 20: 245-254.

23. Popova SN, Bergqvist M, Dimberg A, et al. Subtyping of gliomas of various WHO grades by the application of immunohistochemistry. Histopathology 2014; 64: 365-379.

24. Pekmezci M, Rice T, Molinaro AM, et al. Adult infiltrating gliomas with WHO 2016 integrated diagnosis: additional prognostic roles of ATRX and TERT. Acta Neuropathol 2017; 133: 1001-1016 
25. Hartmann C, Hentschel B, Wick W, et al. Patients with IDH1 wild type anaplastic astrocytomas exhibit worse prognosis than IDH1-mutated glioblastomas, and IDH1 mutation status accounts for the unfavorable prognostic effect of higher age: implications for classification of gliomas. Acta Neuropathologica 2010; 120: 707-718.

26. Kim YH, Nobusawa S, Mittelbronn M, et al. Molecular classification of low-grade diffuse gliomas. Am J Pathol 2010; 177 : 2708-2714.

27. Chen JR, Yao Y, Xu HZ, et al. Isocitrate dehydrogenase (IDH)1/2 mutations as prognostic markers in patients with glioblastomas. Medicine (Baltimore) 2016; 95: e2583.

28. Combs SE, Rieken S, Wick W, et al. Prognostic significance of IDH1 and MGMT in patients with glioblastoma: One step forward, and one step back? Radiat Oncol 2011; 6: 115.

29. Paldor I, Drummond KJ, Kaye AH. IDH1 mutation may not be prognostically favorable in glioblastoma when controlled for tumor location. J Clin Neurosci 2016; 34: 117-120.

30. Kannan K, Inagaki A, Silber J, et al. Whole-exome sequencing identifies ATRX mutation as a key molecular determinant in lower-grade glioma. Oncotarget 2012; 3: 1194-1203.

31. Liu XY, Gerges N, Korshunov A, et al. Frequent ATRX mutations and loss of expression in adult diffuse astrocytic tumours carrying IDH1/IDH2 and TP53 mutations. Acta Neuropathol 2012; 124: 615-625.

32. Reuss DE, Sahm F, Schrimpf D, et al. ATRX and IDH1$\mathrm{R} 132 \mathrm{H}$ immunohistochemistry with subsequent copy number analysis and IDH sequencing as a basis for an "integrated" diagnostic approach for adult astrocytoma, oligodendroglioma and glioblastoma. Acta Neuropathol 2015; 129: 133-146

33. Uppar AM, Sugur H, Prabhuraj AR, et al. H3K27M, IDH1 and ATRX expression in pediatric GBM and their clinical and prognostic significance. Childs Nerv Syst 2019; 35: 1537-1545.

34. Clark AJ, Dos Santos WG, McCready J, et al. Wilms tumor 1 expression in malignant gliomas and correlation of 1 KTS isoforms with p53 status. J Neurosurg 2007; 107: 586-592.

35. Schittenhelm J, Beschorner R, Simon P, et al. Diagnostic value of WT1 in neuroepithelial tumours. Neuropathol Appl Neurobiol 2009; 35: 69-81.

36. Rushing EJ, Sandberg GD, Horkayne-Szakaly I. High-grade astrocytomas show increased Nestin and Wilms's Tumor Gene (WT1) protein expression. Int J Surg Pathol 2010; 18: 255-259.

37. Schmidt MC, Antweiler S, Urban N, et al. Impact of genotype and morphology on the prognosis of glioblastoma. J Neuropathol Exp Neurol 2002; 61: 321-328

38. Bourne TD, Elias WJ, Lopes MB, Mandell JW. WT1 is not a reliable marker to distinguish reactive from neoplastic astrocyte populations in the central nervous system. Brain Pathol 2010; 20: 1090-1095.

39. Rauscher J, Berchorner R, Gierke M, et al. WT1 expression increases with malignancy and indicates unfavourable outcome in astrocytoma. J Clin Pathol 2014; 67: 556-561.

40. Schwab DE, Lepski G, Borchers C, et al. Immunohistochemical comparative analysis of GFAP, MAP-2, NOGO-A, OLIG-2 and WT-1 expression in WHO 2016 classified neuroepithelial tumours and their prognostic value. Pathol Res Pract 2018 214: $15-24$

41. Camacho-Urkaray E, Santos-Juanes J, Gutiérrez-Corres FB, et al. Establishing cut-off points with clinical relevance for bcl-2, cyclin D1, p16, p21, p27, p53, Sox11 and WT1 expression in glioblastoma - a short report. Cell Oncol 2018; 41: 213-221.

42. Rauscher J, Beschorner R, Gierke M, et al. WT1 expression increases with malignancy and indicates unfavourable outcome in astrocytoma. J Clin Pathol 2014; 67: 556-561.

43. Watanabe K, Tachibana O, Sata K, et al. Overexpression of the EGF receptor and p53 mutations are mutually exclusive in the evolution of primary and secondary glioblastomas. Brain Pathol 1996; 6: 217-223.
44. Simmons ML, Lamborn KR, Takahashi M, et al. Analysis of complex relationships between age, p53, epidermal growth factor receptor, and survival in glioblastoma patients. Cancer Res 2001; 61: 1122-1128.

45. Smith JS, Tachibana I, Passe SM, et al. PTEN mutation, EGFR amplification, and outcome in patients with anaplastic astrocytoma and glioblastoma multiforme. J Natl Cancer Inst 2001; 93: $1246-1256$

46. Ohgaki H, Dessen P, Jourde B, et al. Genetic pathways to glioblastoma: a population-based study. Cancer Res 2004; 64: 6892-6899.

47. Montgomery RM, Queiroz LS, Rogerio F. EGFR, p53, IDH1 and MDM2 immunohistochemical analysis in glioblastoma: therapeutic and prognostic correlation. Arq Neuropsiquiatr 2015; 73: 561-568.

\section{Address for correspondence}

\section{Gülsün Gülten}

Pathology Department

Pamukkale University Faculty of Medicine

Denizli, Turkey

e-mail: dr.gulsun_88@hotmail.com 